\title{
COMUNICAÇÃO
}

\section{ENCONTRO DE PANSTRONGYLUSMEGISTUSEM ECÓTOPO ARTIFICIAL: DOMICILIAÇÃO OU MERA VISITAÇÃO?}

\author{
Carlos Nascimento, Ana M. Marassá, Izilda Curado e Roxane M.F. Piazza
}

\begin{abstract}
No combate à doença de Chagas no Brasil, foi utilizado como primeira medida o controle químico, erradicando o Triatoma infestans, o mais importante vetor, tal combate vem favorecendo o aparecimento de vetores secundários, como Triatoma sordida and Panstrongylus megistus, espécies que podem eventualmente ser encontradas no domicílio, como os triatomíneos provenientes de Bernardino de Campos e Sete Barras por nós examinados que foram encontrados no domicílio e positivos para o T. cruzi, sugerindo que, apesar da doença de Chagas estar controlada no Estado de São Paulo, existe a necessidade de aprimorar os conhecimentos sobre o comportamento destes vetores para que mudanças nas medidas de controle sejam introduzidas.
\end{abstract}

Palavras-chaves: P. megistus. Domiciliação. Trypanosoma cruzi.

A doença de Chagas, primitivamente uma enzootia, passou a constituir-se em problema de saúde humana, a partir da domiciliação dos vetores. A degradação do ambiente natural, com o deslocamento de triatomíneos de seus ecótopos silvestres primitivos, é que determinou a transmissão domiciliar da doença. Além dos determinantes ecológicos, há aqueles de natureza econômica e social, expressos pelas más condições de habitação. É doença de espaços abertos, naturais ou decorrentes da ação antrópica, e é doença produzida pela pobreza.

A epidemiologia da doença e as tecnologias disponíveis limitam o controle da transmissão natural à intervenção sobre os vetores, como alternativa prática, ações que evitem o contato dos mesmos com o homem no ambiente domiciliar, de um lado, o grande número de reservatónios silvestres e, de outro, a inexistência de drogas que possam ser usadas em larga escala, bem como a inexistência de imunizantes fazem impossível o controle na perspectiva de esgotamento das fontes de infecção. $\mathrm{O}$ ideal seria que houvesse uma melhoria dos padrões de habitação, que dificultasse ao máximo a domiciliação dos vetores.

\footnotetext{
Laboratório de Imunoparasitologia e Laboratório de Entomologia, Instituto Butantan, São Paulo, SP, Brasil.

Trabalho financiado pela Fundação Butantan.

Endereço para correspondência: Dra Roxane M.F. Piazza. Laboratório de Imunoparasitologia/Instituto Butantan, Av. Vital Brazil 1500, 05503-900 São Paulo, SP, Brasil.

Fax: (011) 815-1505.E.mail: roxane@usp.br.

Recebido para publicação em 18/06/96.
}

O programa de controle de doenca de Chagas no Brasil instituído, a partir de 1975, teve como prioridade o controle da transmissão primária vetorial e adotou como metodologia, o controle químico do Triatoma infestans, o mais importante vetor. Os indicadores entomológicos de uso corrente revelam a virtual eliminação de T. infestans, espécie introduzida no país e estritamente domiciliada. Inquéritos sorológicos em grupos etários jovens confirmam o impacto sobre a transmissão. Dados de morbidade, e mesmo de mortalidade, são também fortemente sugestivos de que o controle vetorial determinou importante redução no número de casos e de óbitos atribuídos à doença de Chagas4. Como parte da rotina de operações deste programa em 1983, quando concluídos os levantamentos entomológicos, somente cinco espécies se julgava importantes na transmissão ao homem (T. infestans, P. megistus, T. brasiliensis, T. pseudomaculata e T. sordida).

No Estado de São Paulo, a Superintendência de Controle de Endemias (SUCEN) considera que a transmissão da doença de Chagas pelo T. infestans (principal vetor da doença) tenha sido interrompida no início da década de 70 . Se houve uma transmissão residual, em níveis muito baixos, além desse período, ela deve ter ocorrido numa área restrita do Estado, que engloba municípios da região de Sorocaba. Os levantamentos sorológicos de prevalência de infecção humana realizados no Estado indicam que a doença de Chagas cessou antes de 19745 .

Fato preocupante é o de que, nas áreas limpas de T. infestans, podem permanecer as 
Comunicação. Nascimento C, Marassá AM, Curado I, Piazza RMF. Encontro de Panstrongylus megistus em ecótopo artificial: domiciliação ou mera visitação? Revista da Sociedade Brasileira de Medicina Tropical 30:333-336, jul-ago, 1997.

espécies de T. sordida e P. megistus, que não apresentam a mesma suscetibilidade da primeira aos inseticidas normalmente usados no controle, podendo-se manter apenas níveis de infestação teoricamente incompatíveis com a transmissão. Esta situação tem sido motivo de preocupação por parte da SUCEN, diante da perspectiva de que o vazio ecológico determinado pela eliminação do T. infestans fosse ocupado por esses vetores6.

As informações acima descritas e o afluxo de indíviduos ao Laboratório de Entomologia do Instituto Butantan, trazendo exemplares de triatomíneos encontrados em seus domicílios em diferentes regiões do Estado de São Paulo e Minas Gerais para identificação, levaram-nos à presente comunicação, pois, nossos dados parecem comoborar os de Wanderley6 sobre domiciliação de vetores secundários. Os exemplares foram identificados no período de 1991 a 1996 como P. megistus (52 exemplares), P. geniculatus (2 exemplares), T. sordida (4 exemplares), T. tibiamaculata (11 exemplares), Rhodnius domesticus (3 exemplares) e T. vitticeps (1 exemplar). Após a identificação, todos aqueles que chegaram vivos foram investigados quanto à presença de formas flageladas nas fezes e urina.

Em outubro de 1995 foram trazidos 14 exemplares de triatomíneos, adultos machos e fêmeas e ninfas, provenientes do município de Bemardino de Campos, distante cerca de $350 \mathrm{~km}$ da capital e em abril de 1991 um exemplar desse mesmo inseto proveniente do município de Sete Barras, distante cerca de $230 \mathrm{~km}$ da capital do Estado de São Paulo (Figura 1). Estes insetos foram coletados pelos proprietários das casas em que foram encontrados, sendo

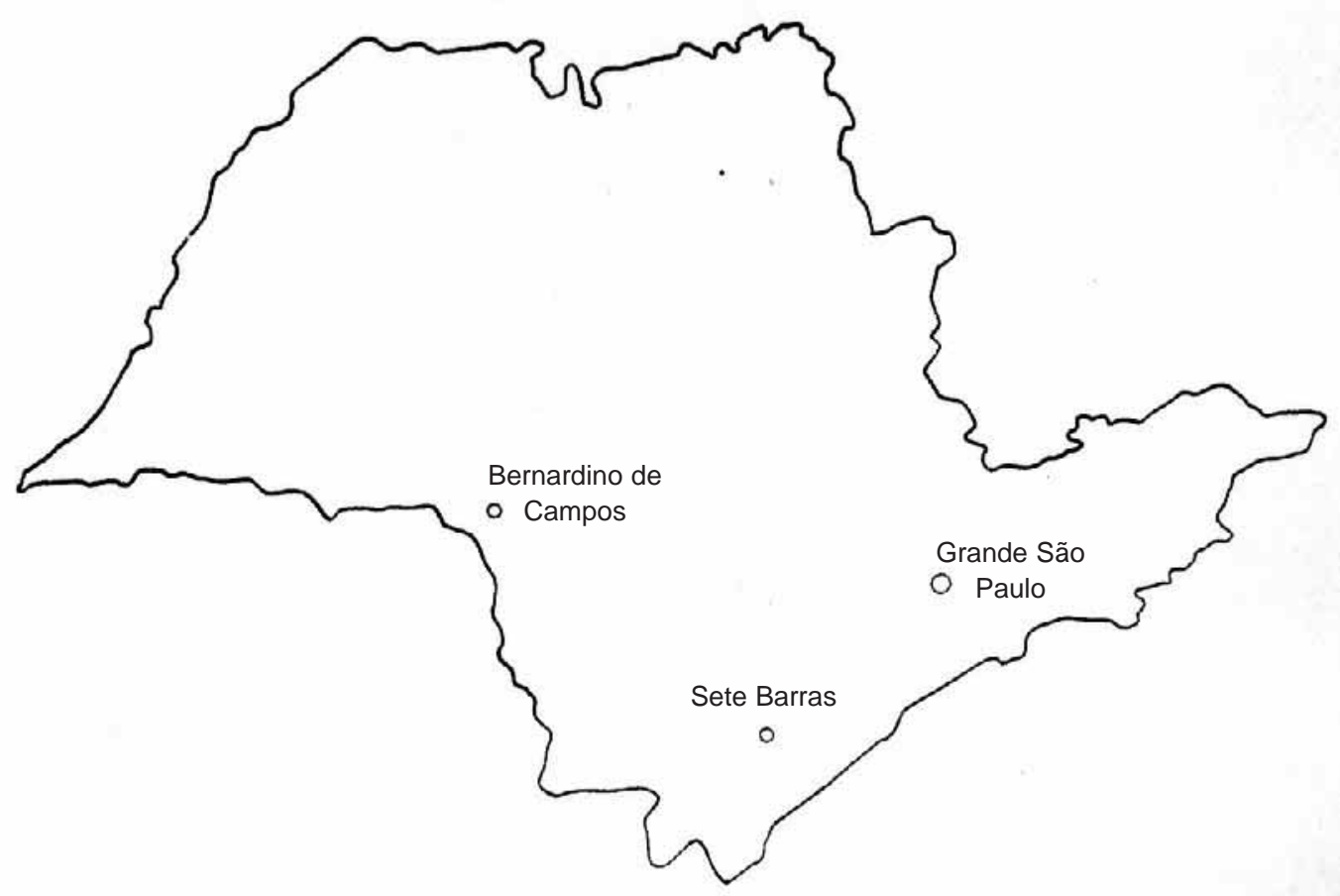

Figura 1 - Mapa do Estado de São Paulo indicando as áreas onde foram encontrados os triatomíneos examinados. Bernardino de Campos e Sete Barras.

que o morador de Bernardino de Campos referiu-se à existência de um ninho de gambá no teto da moradia. Todos eles foram identificados como pertencentes à espécie Panstrongylus megistus.
Dos 14 P. megistus provenientes de Bernardino de Campos, somente 5 deles puderam ser examinados quanto à presença de formas flageladas em suas fezes, bem como o exemplar de Sete Barras, sendo todos positivos. 
Comunicação. Nascimento C, Marassá AM, Curado I, Piazza RMF. Encontro de Panstrongylus megistus em ecótopo artificial: domiciliação ou mera visitação? Revista da Sociedade Brasileira de Medicina Tropical 30:333-336, jul-ago, 1997.

O material obtido por compressão abdominal, foi inoculado intraperitonealmente em camundongos Balb/C machos, com idade entre 4 e 6 semanas. A avaliação da evolução da parasitemia foi realizada 3 vezes por semana, utilizando-se o método descrito por Brener1. A análise microscópica das lâminas com sangue de camundongos inoculados com cada um dos isolados de Bernardino de Campos foi negativa para presença de parasitas em 3 experimentos independentes. Após 30 dias sem aparecimento de parasitas no sangue circulante dos camundongos, foi feita punção cardíaca em cada um deles. O sangue obtido foi diluído em citrato de sódio 3,8\%, sendo imediatamente semeado em meio LT (liver infusion tryptose) 2 , onde após alguns dias foram encontradas formas tripomastigotas e epimastigotas morfologicamente iguais ao $\mathrm{T}$. cruzi. Estes dados sugerem que tais isolados desenvolvem parasitemia sub-patente em camundongos.

Por outro lado, a cepa de Sete Barras, apresenta um período pré-patente de 12 dias, com mortalidade de $100 \%$ dos animais inoculados e alta virulência (Figura 2). Tais dados mostram a importância do conhecimento

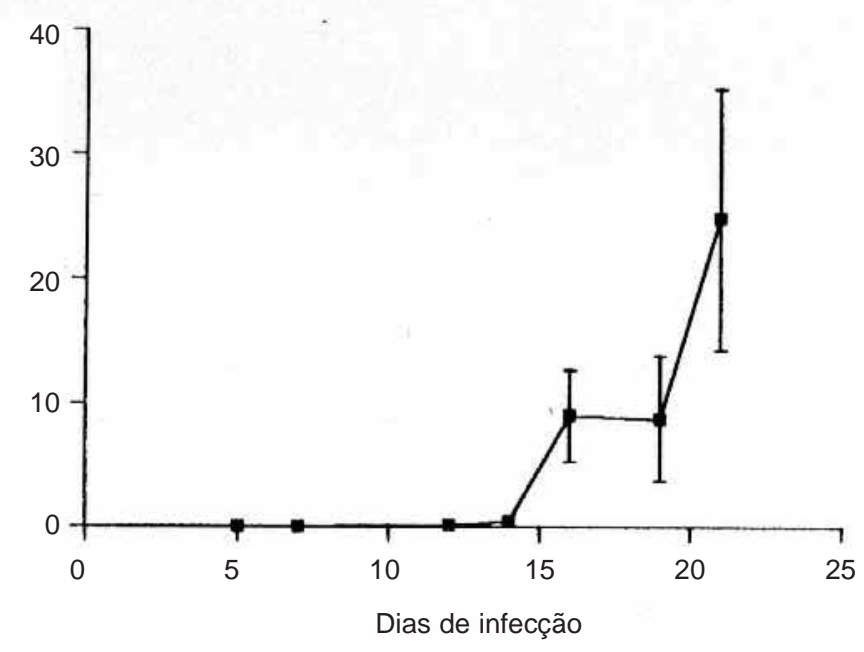

Figura 2 - Curva de parasitemia obtida com 5 camundongos Balb/C machos inoculados com 5000 formas tripomastigotas sanguíneas da cepa Sete Barras. Mediana de 4 experimentos independentes.

dos vetores bem como das cepas de T. cruzi que estes albergam.O P. megistus, espécie que identificamos, poderia ser considerada hoje a mais importante espécie vetora da doença de Chagas no Brasil, devido à sua ampla distribuição geográfica. Depois de T. infestans, esta é a espécie com maior capacidade de domiciliação. É um triatomíneo que apresenta diferentes comportamentos ecológicos nas regiões nordeste e sudeste do país. Essa espécie não é fácil de controlar por programas convencionais de aplicação de inseticidas. No controle de espécies que se colonizam no peridomicílio, deve ser esperada menor resposta às ações de inseticidas, na medida em que este ambiente propicia uma degradação mais rápida do produto, diminuindo o seu poder residual. Neste contexto a vigilância deve ser permanente, programando e estimulando a ação participativa da população na denúncia de focos intradomiciliares6.

Em nosso caso, a princípio, parece-nos que pelo fato dos exemplares de P.megistus terem sido encontrados dentro do domicílio em diferentes estágios evolutivos, inclusive com a presença de ninho de gambá no interior da moradia em que foram coletados, tratar-se-ia não apenas de visitação destes insetos ao domicilio, mas sim de sua colonização. Caso nossa hipótese seja verdadeira, a domiciliação desta espécie pode estar ocorrendo com certa 
Comunicação. Nascimento C, Marassá AM, Curado I, Piazza RMF. Encontro de Panstrongylus megistus em ecótopo artificial: domiciliação ou mera visitação? Revista da Sociedade Brasileira de Medicina Tropical 30:333-336, jul-ago, 1997.

freqüência por várias localidades do Estado. Além disto, verificamos que os camundongos inoculados com a cepa de Sete Barras, apresentaram paralisia do trem posterior bem como mortalidade de 100\% destes animais. Desta forma, tais insetos, além de provavelmente estarem domiciliados, ainda foram capazes de albergar uma cepa classificada como de alta virulência como definido por Schlemper e cols 3 .

Assim sendo, apesar da doença de Chagas estar controlada no Estado de São Paulo, nossos dados alertam para a necessidade de que sejam envidados esforços para que se obtenha um melhor conhecimento do comportamento dos vetores. Para tanto, é necessário reconhecer que o controle não pode ser reduzido ao combate químico, a fim de que possíveis mudanças que contribuam para o aperfeiçoamento do mesmo, possam ser incorporadas.

\section{SUMMARY}

As a first measure of Chagas' disease control in Brazil with chemical elimination of the most important vector of the disease, Triatoma infestans was removed. Attention is now being paid to Triatoma sordida and Panstrongylus megistus. That species can eventualy be found inside houses, as happened with the specimens we examined from Bernardino de Campos and Sete Barras, all of them infected by Trypanosoma cruzi. These data suggest that a better knowledge about the behavior that species is needed to introduce changes in the control measures .

Key-words: Chagas' disea se. P. megistus domiciled. Trypanosoma cruzi.

\section{AGRADECIMENTOS}

À Prof ${ }^{\mathrm{a}}$. Dr ${ }^{\mathrm{a}}$ Judith K. Kloetzel pelas sugestões, críticas e revisão deste manuscrito e ao Manoel Machado da Cunha Neto pela arte gráfica.

\section{REFERÊNCIAS BIBLIOGRÁFICAS}

1. Brener Z. Contribuição ao estudo da terapêutica experimental da doença de Chagas. Tese de livredocência, Universidade Federal de Minas Gerais, Minas Gerais, MG, 1961.

2. Camargo EP. Growth and differentiation in Trypanosoma cruzi: I. Origin of metacyclic trypanosomes in liquid media. Revista do Instituto de MedicinaTropical de São Paulo 6:93100, 1964.

3. Schlemper Jr BR, Ávila CM, Coura JR, Brener Z. Cause of infection and histopathological lesions in mice infected with seventeen $T$. cruzi strains isolated from chronic patients. Revista da Sociedade Brasileira de Medicina Tropical 16:2330, 1983.
4. SilveiraAC, Rezende DF. Epidemiologia e controle da transmissão vetorial da doença de Chagas no Brasil. Revista da Sociedade Brasileira de Medicina Tropical 27 (supl III):11-22, 1994.

5. Wanderley DMV. Controle de Triatoma infestans no Estado de São Paulo. Revista da Sociedade Brasileira de Medicina Tropical 26 (supl III):1725, 1993.

6. Wanderley DMV. Vigilância do risco de transmissão da doença de Chagas por vetores secundários no Estado de São Paulo. Revista da Sociedade Brasileira de Medicina Tropical 26 (supl II):38-40, 1993. 